\title{
Molecular Genetics of Human Personality Traits for Psychiatric, Behav- ioral, and Substance-Related Disorders
}

\author{
Eugene $\operatorname{Lin}^{*, 1}$ and Po See Chen*,2,3

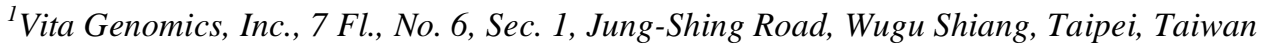 \\ ${ }^{2}$ Department of Psychiatry, National Cheng Kung University, Tainan, Taiwan \\ ${ }^{3}$ National Cheng Kung University Hospital and Dou-Liou Branch, Taiwan
}

\begin{abstract}
The investigation of personality genetics had received much attention since the three seminal reports showing an association between genes and personality traits in the general population. Accumulating evidences suggested that personality traits have significant genetic components. Although currently available data are not enough for proof, more and more genetic variants associated with personality traits are being discovered. In this paper, we review related studies of gene polymorphisms and human personality traits for psychiatric, behavioral, and substance-related disorders. First, we briefly describe the commonly-used self-reported temperament measures that define personality dimensions. Then, we summarize the characteristics of the candidate genes for personality traits, and investigate gene variants which have been suggested to be linked with personality traits for individuals with psychiatric, behavioral, and substance-related disorders.
\end{abstract}

Keywords: Molecular genetics, personality, psychiatric disorders, temperament measures.

\section{INTRODUCTION}

The investigation of personality genetics had received much attention since the three seminal reports [1-3] in 1996 showing an association between genes and personality traits in the general population. Accumulating evidences suggested that personality traits have significant genetic components [4-7]. Although currently available data are not enough for proof, more and more genetic variants associated with personality traits are being discovered [4-7]. Personality traits can be measured by several self-reported questionnaires including the Tridimensional Personality Questionnaire (TPQ) [8], the Temperament and Character Inventory (TCI) [9], the Revised NEO Personality Inventory (NEO-PI-R) [10], the Eysenck Personality Questionnaire (EPQ) [11,12], and the Zuckerman-Kuhlman Personality Questionnaire (ZKPQ) [13].

Related studies have identified the serotonin transporter gene (SLC6A4) polymorphisms as genetic markers that are correlated with personality traits, such as NEO-PI-R neuroticism and TCI harm avoidance [14-16]. A common SLC6A4 gene polymorphism is the serotonin transporter gene-linked polymorphic region (5-HTTLPR) variant. A recent metaanalysis observed a significant association between 5HTTLPR and NEO-PI-R neuroticism, but not with TCI/TPQ harm avoidance or EPQ neuroticism [16]. This result was consistent with previous reports which indicated that there was evidence of association between NEO-PI-R neuroticism

*Address correspondence to these authors at the Vita Genomics, Inc., 7 Fl., No. 6, Sec. 1, Jung-Shing Road, Wugu Shiang, Taipei, Taiwan; Tel: (+ 886) 2-8976-9123 ext, 7751; Fax: (+ 886) 2-8976-9523;

E-mail: eugene.lin@vitagenomics.com

Department of Psychiatry, National Cheng Kung University, Tainan, Taiwan; Tel: (+ 886) 6-235-3535 ext, 5190; Fax: (+ 886) 6-275-9259;

E-mail: chenps@mail.ncku.edu.tw and 5-HTTLPR but not other anxiety-related personality traits $[14,15]$.

We reviewed related studies of gene polymorphisms and human personality traits for psychiatric, behavioral, and substance-related disorders. First, we briefly describe the commonly-used self-reported temperament measures that define personality dimensions. Then, we summarize the characteristics of the candidate genes for personality traits, and investigate gene variants which have been suggested associated with personality traits for individuals with psychiatric, behavioral, and substance-related disorders, including alcoholism, anxiety-depression alcoholism, antisocial personality disorder with alcoholism, borderline personality disorder, binge eating disorder, bulimia nervosa, internet addiction, mood disorders, and nicotine dependence. One of the intentions of these previous studies was that our knowledge of the genetics of personality may enhance our understanding of the genetic basis of psychiatric, behavioral, and substancerelated disorders. Moreover, examination of subjects in specific populations with these disorders may improve our understanding of the genetic basis of personality in the general population.

\section{SELF-REPORTED TEMPERAMENT MEASURES}

\subsection{Tridimensional Personality Questionnaire (TPQ)}

Based on a general biosocial theory of personality, Cloninger proposed that the TPQ defines three dimensions of personality in terms of the basic stimulus-response characteristics of novelty seeking, harm avoidance, and reward dependence [8]. Novelty seeking tends to be associated with low basal dopaminergic activity and is related to a tendency toward exploratory behavior in search of novel stimuli, impulsive responding, and extravagance in approach to cues of reward $[9,17]$. Harm avoidance tends to be associated with 
high serotonergic activity and involves a tendency to overrespond to aversive stimuli, worry about future problems, fear uncertainty, and act shy around strangers $[9,18]$. Reward dependence tends to be associated with low noradrenergic activity and is characterized by a tendency toward sentimentality, social sensitivity, attachment, and dependence on approval by others $[9,19]$.

\subsection{Temperament and Character Inventory (TCI)}

The TPQ is subsequently evolved into the TCI which describes a self-reported measure of seven personality dimensions, including four dimensions of temperament (novelty seeking, harm avoidance, reward dependence and persistence) and three dimensions of character (self-directedness, cooperativeness, self-transcendence) [9]. The TCI has 226 items and has an abbreviated version that employs a truefalse format with 125 items.

\subsection{Revised NEO Personality Inventory (NEO-PI-R)}

The NEO-PI-R is a 240-question measure of the Five Factor Model: neuroticism, extraversion, openness to experience, agreeableness, and conscientiousness [10]. Traits in the neuroticism domain describe different ways of reacting emotionally to distressing circumstances. The extraversion domain is concerned with energy and enthusiasm. The facets of the openness to experience domain are associated with intellectual curiosity and aesthetic sensitivity. The agreeableness domain measures traits related to styles of interpersonal interaction. Traits in the conscientiousness domain reflect differences in motivation and persistence.

A study showed that The TPQ/TCI significantly correlated with the NEO-PI-R [20], demonstrating that TPQ/TCI novelty seeking is correlated to NEO-PI-R extraversion and openness to experience and negatively to conscientiousness. Furthermore, TPQ/TCI harm avoidance is strongly related positively with NEO-PI-R neuroticism and negatively to extraversion. In addition, TPQ/TCI reward dependence is primarily correlated with NEO-PI-R extraversion and secondarily with openness to experience. Finally, TPQ/TCI persistence is highly related to conscientiousness.

\subsection{Eysenck Personality Questionnaire (EPQ)}

The EPQ conceptualizes personality as three categories of temperament: extraversion (impulsiveness and sociability), psychoticism (vulnerability to psychoses) and neuroticism (emotional instability) [11,12]. The EPQ has 100 items and has a 48-item short scale questionnaire.

A study suggested that TPQ/TCI harm avoidance represents a 45 degree rotation of EPQ extraversion and neuroticism most likely [21]. Moreover, TPQ/TCI novelty seeking appears to be further rotated into EPQ pychoticsm space. However, TPQ/TCI reward dependence is not equivalent to EPQ psychoticism, showing that TPQ/TCI reward dependence is negatively related with EPQ psychoticism modestly.

\subsection{Zuckerman-Kuhlman Personality Questionnaire (ZKPQ)}

In order to narrow the scope of study on risk-taking and personality, the ZKPQ assesses personality along for five dimensions, including impulsive sensation seeking (which has two subscales: impulsivity and sensation seeking), neu- roticism-anxiety, aggression-hostility, activity (formed by two subscales: work effort and general activity), and sociability (made up of two subscales: isolation intolerance and liking of parties and friends) [13]. The ZKPQ is an 89-item self-reported measure and is an extension of the EPQ three dimensions of personality.

\section{PERSONALITY GENETICS FOR PSYCHIATRIC DISORDERS}

In this paper, we review the following candidate genes that may be strongly associated with personality traits for psychiatric, behavioral, and substance-related disorders: dopamine D2 receptor (DRD2), dopamine D4 receptor (DRD4), 5-hydroxytryptamine receptor 2A (HTR2A; serotonin receptor 2A), monoamine oxidase A (MAOA), SLC6A4, and tryptophan hydroxylase 1 (TPH1).

\subsection{Dopamine Receptors}

Dopamine receptors play a key role in many processes, including motor behavior, motivation, and working memory [22]. DRD2 and DRD4 receptors are subtypes of dopamine receptors, and their common gene polymorphisms are DRD2 TaqIA polymorphism and DRD4 exon III polymorphism, respectively. Evidence showed that they contribute to personality traits in the general population [7].

\subsubsection{Anxiety-Depression Alcoholism}

In a study of Lin and colleagues, they investigated a Taiwanese population of 87 anxiety-depression alcohol dependents and 46 pure alcohol dependents, to see whether the TaqIA polymorphism of the DRD2 gene is involved in TPQ novelty seeking and harm avoidance [23]. DRD2 TaqI A1(+) allele (A1/A1 or A1/A2 genotypes) was found to be associated with TPQ novelty seeking in anxiety-depression alcohol dependents [23].

Anxiety-depression alcohol dependence was defined as a genetically specific subtype of alcoholism [24]. Four homogeneous types of alcoholism have been suggested, including the depressed/anxious type, the chronic/severe type, the mildly affected type and the antisocial type [25]. Anxiety disorders represent one of the most common mental illnesses and are complex diseases with both genetic and environmental factors affecting their predisposition [26]. Recent data revealed that the genetic variant of the DRD2 gene was associated with anxiety-depression alcohol dependence [27].

\subsubsection{Antisocial Personality Disorder with Alcoholism}

In a Taiwanese population of antisocial personality disorder, 43 with alcoholism and 84 without alcoholism, Wu and colleagues examined the DRD2 gene and the SLC6A4 5-HTTLPR variant on TPQ novelty seeking [28]. They found evidence for an interaction between DRD2 TaqI A1+ (including A1/A1 or A1/A2) and 5-HTTLPR short/short genotype in the novelty seeking scores, and found significant difference in 5-HTTLPR polymorphisms between antisocial alcoholics and antisocial non-alcoholics after stratification of DRD2 TaqIA genotypes [28].

Antisocial personality disorder is a mental disorder defined by a pervasive pattern of deception, manipulation, disregard for the rights of others, and a lack of remorse for their behavior that begins in childhood or early adolescence [29]. 
Genetic studies showed evidence of genetic influences on personality disorder, such as antisocial personality disorders, borderline personality disorders, schizotypal personality disorders [30-32]. A recent study suggested that the possible interactions between the MAOA and DRD2 genes might be related to antisocial personality disorder with alcoholism among Taiwanese [33]. Future research should further investigate the contributions of the MAOA gene to personality traits in antisocial personality disorder with alcoholism.

\subsubsection{Binge Eating Disorder}

Davis and colleagues compared participants with binge eating disorder $(n=56)$ to normal-weight $(n=59)$ and obese controls $(n=51)$ on reward sensitivity and genotyped six markers (TaqIA, -141C Ins/Del, -241A/G, Taq1D, C957T, and rs4648317) of the DRD2 dopamine receptor gene [34]. Two personality measures of reward sensitivity were employed, including the Behavioral Inhibition System/ Behavioral Activation System (BIS/BAS) Scales [35] and the Sensitivity to Punishment and Sensitivity to Reward Questionnaire (SPSRQ) [36]. They reported that binge eating disorder and obese participants had greater reward sensitivity than normal-weight controls, but only among those carrying the A1 allele of the TaqIA marker [34].

Binge eating disorder is a provisional eating disorder diagnosis, which core symptom is recurrent binge eating $[37,38]$, and the episode requires consumption of an unusually large amount of food and a sense of being out of control. In addition, individuals with binge eating disorder do not regularly engage in compensatory behaviors. Recent studies suggested that the 5-HTTLPR [39] and ghrelin/obestatin prepropeptide (GHRL) [40] may contribute to the genetic susceptibility to the disorder. Future research should further investigate the contributions of the SLC6A4 and GHRL genes to personality traits in binge eating disorder.

\subsubsection{Mood Disorders}

In a study of 73 major depressive disorder and 134 bipolar disorder patients, Serretti and colleagues tested the hypothesis that the exon III variant in the DRD4 gene could influence personality traits in the patients of mood disorders [41]. Genotypes with the long repeat of DRD4 exon III polymorphism were marginally associated with low TCI harm avoidance [41].

Depression and bipolar disorder are the two major types of mood disorders, in which the emotional mood of a patient is distorted or inappropriate to the circumstances [42]. Owing to the fact that personality is itself a complex trait, its use as an endophenotype for genetic studies of bipolar disorder has certain limitations [43]. Newer hypotheses of depression neurobiology suggest an association of major depressive disorder with polymorphisms in the brain-derived neurotrophic factor (BDNF) gene [44]. Future research should further study the contributions of the BDNF gene to personality traits in major depressive disorder.

\subsection{HTR2A}

Serotonin receptors are receptors for the neurotransmitter serotonin, which is also known as 5-hydroxytryptamine. HTR2A receptors are a subtype of serotonin receptors and play an important role in embryogenesis and in the periphery [45]. It was reported that there was a credible association between the HTR2A gene and personality traits in the general population [7].

\subsubsection{Borderline Personality Disorder}

Based on a Caucasian sample of 111 patients with borderline personality disorder and 287 healthy controls, $\mathrm{Ni}$ and colleagues tested the association between the HTR2A gene and personality traits in borderline personality disorder [46]. Among four polymorphisms (rs6313, rs4941573, rs2296972, and rs6314) of the HTR2A gene, the $\mathrm{C}$ allele of rs6313 and the A allele of rs4941573 were found to be associated with a higher NEO-PI-R extraversion score, indicating that there were significant associations between the HTR2A gene and personality traits in the patients with borderline personality disorders [46].

Borderline personality disorder, which begins in early adulthood, is a chronic and severe mental disorder characterized by a pervasive pattern of instability in affect regulation, interpersonal relationships, self-image, and impulse control [47]. The SLC6A4 and MAOA genes have been suggested to be involved in the development of it [48]. A recent study demonstrated that there were gender differences in TCI novelty seeking in borderline personality disorder [49]. In future work, the contributions of the MAOA gene to personality traits in borderline personality disorder should be further investigated.

\subsection{MAOA}

The MAOA gene is located on the short arm of the $\mathrm{X}$ chromosome and encodes the MAOA enzyme that degrades amine neurotransmitters such as dopamine, norepinephrine, and serotonin [50]. The functional MAOA-upstream variable number of tandem repeats (MAOA-uVNTR) polymorphism in the MAOA gene promoter commonly consists of 3 (3allele) or 4 (4-allele) copies of a 30-bp sequence, or rarely 2 (2-allele) or 3 copies plus the first 18 -bp of the same $30-b p$ sequence ( $3 \mathrm{a}$-allele), or 5 copies (5-allele). It was suggested that the MAOA gene was found to be associated with personality traits in the general population [7].

\subsubsection{Mood Disorders}

Serretti and colleagues [41] tested the uVNTR variant in the MAOA gene to see if it influences personality traits in the patients of mood disorders or not. The long MAOA allele was found to be associated with decreased TCI persistence scores among female patients [41].

\subsection{SLC6A4}

The SLC6A4 gene encodes an integral membrane protein that transports the neurotransmitter serotonin from synaptic spaces into presynaptic neurons, terminates the action of serotonin, and recycles it into the neurotransmitter pool [51]. The common SLC6A4 gene polymorphisms are the short and long alleles of the 5-HTTLPR variant and alleles of the variable number tandem repeats (VNTR) region in the second intron. There are three possible alleles of VNTR, including 9 repeats $(345 \mathrm{bp}), 10$ repeats $(360 \mathrm{bp})$, and 12 repeats (390 bp), and the possible genotypic combinations are as follows: 9/10, 9/12,10/10,10/12 and 12/12. Anxiety-related personality traits, mood disorders, antidepressants response, alcoholism, smoking, and eating disorders have been found to be related to the SLC6A4 variants [52,53]. 


\subsubsection{Alcoholism}

In a study on German alcohol dependents $(\mathrm{n}=368)$, Koller and colleagues [54] hypothesized that the 5-HTTLPR and VNTR variants of the SLC6A4 gene are associated with TCI harm avoidance. An association between short/12-repeat haplotype of SLC6A4 and low level of TCI harm avoidance was found [54]. Furthermore, no significant association was found between TCI harm avoidance and the SLC6A4 5HTTLPR variant as well as between TCI harm avoidance and the SLC6A4 VNTR variant [54].

Conversely, in a study of 124 subjects seeking inpatient treatment for primary alcohol dependence, Wiesbeck and colleagues reported that the SLC6A4 5-HTTLPR variant was associated with TCI harm avoidance in the group [55]. The conflicting results between this work and the report [54] by Koller and colleagues may be due to the differences in sample sizes, different ethnicities, or different study designs. The studies conducted on small patient populations may also have biased a particular result.

Alcoholism is a chronic disease characterized by alcohol abuse despite recurrent adverse consequences [56]. Recent evidence supports genetic associations with alcoholism of the glutamate receptor ionotropic N-methyl D-aspartate 2A (GRIN2A) gene [57] and MAOA genes [27]. In future work, the contributions of the GRIN2A and MAOA genes to personality traits in alcohol dependence should be further tested.

\subsubsection{Anxiety-Depression Alcoholism}

Lin and colleagues [23] assessed whether the SLC6A4 5HTTLPR variant is involved in TPQ novelty seeking and harm avoidance, and found that the short/short genotype of the SLC6A4 5-HTTLPR variant was associated with TPQ novelty seeking in anxiety-depression alcohol dependents [23].

\subsubsection{Borderline Personality Disorder}

Pascual and colleagues investigated the association between the SLC6A4 5-HTTLPR and VNTR polymorphisms and personality traits in their study of 65 patients with borderline personality disorder [58]. They reported that patients with long allele (long/short or long/long) in the 5-HTTLPR polymorphism of the SLC6A4 gene showed lower scores on the subscale of the ZKPQ liking of parties and friends [58]. Furthermore, patients with the allele with 10 repeat of the SLC6A4 VNTR polymorphism were found to show lower scores in the ZKPQ impulsivity, sensation seeking and in the subscale liking of parties and friends [58].

\subsubsection{Bulimia Nervosa}

Monteleone and colleagues [59] tested whether the 5HTTLPR polymorphism of the SLC6A4 gene is related to TCI harm avoidance or not. In 125 female Caucasian patients with bulimia nervosa and 94 healthy controls, they found that patients with bulimia nervosa carrying at least one copy of the short allele have significantly higher mean TCI harm avoidance score than those with the long/long genotype [59].

The TPH1 gene codes for tryptophan hydroxylase, the rate-determining enzyme in serotonin biosynthesis [60]. Likewise, Monteleone and colleagues [61] tested whether A218C polymorphism of the TPH1 gene is related to TCI harm avoidance. In their study of 180 female Caucasians (91 patients with bulimia nervosa and 89 healthy controls), they demonstrated that bulimic women with the AA genotype of the TPH1 gene exhibited a more severe binge eating behavior and higher TCI harm avoidance scores than those with CC genotype [61]. Together with the similar study [59] previously discussed, the findings of these two studies support the idea that the SLC6A4 5-HTTLPR polymorphism and the TPH1 A218C polymorphism are involved in predisposing bulimic patients to a more disturbed eating behavior and higher TCI harm avoidance [59,61].

Bulimia nervosa is an eating disorder characterized by recurrent binge eating, which is followed by compensatory behaviors, such as self-induced vomiting, fasting, and overexercising $[38,62]$. A recent study revealed that the GHRL gene is associated with susceptibility to bulimia nervosa [63]. In future work, the contributions of the GHRL gene to personality traits in bulimia nervosa should be further assessed.

\subsubsection{Internet Addiction}

Internet addiction or excessive internet use is defined as uncontrollable and damaging use of the internet and has been compared to other addictions such as pathological gambling [64]. In a Korean population of 91 male adolescents with excessive internet use and 75 healthy controls, Lee and colleagues conducted group comparisons on the 5-HTTLPR polymorphism of the SLC6A4 gene with respect to TCI novelty seeking and harm avoidance [65]. It was found that participants with excessive internet use with 5-HTTLPR short/short genotype showed higher TCI harm avoidance scores than the ones with the other allele variants [65].

\subsubsection{Mood Disorders}

Serretti and colleagues [41] further tested the hypothesis that the variants in the SLC6A4 gene could influence personality traits in the patients of mood disorders. It was observed that the 5-HTTLPR short/short genotype frequency was associated with low TCI novelty seeking scores [41].

Furthermore, based on a sample of 251 participants, another study observed that EPQ neuroticism mediates the association between the SLC6A4 5-HTTLPR polymorphism and lifetime major depression [66].

\subsubsection{Nicotine Dependence}

Nicotine dependence is the physical vulnerability of human body to the chemical nicotine, which is a psychoactive ingredient in tobacco significantly contributing to the harmful tobacco smoking habit [67]. In a study of personality genetics, Kremer and colleagues recruited 244 smokers and examined the association of smoking with the 5-HTTLPR and VNTR polymorphisms of the SLC6A4 gene [68]. It was observed that there was a weak association between TPQ novelty seeking and the VNTR polymorphism as well as between TPQ reward dependence and the 5-HTTLPR polymorphism [68].

Recent studies have reported genetic associations with nicotine dependence of the following genes: BDNF $[69,70]$, cholinergic receptor nicotinic alpha 4 (CHRNA4) [71-73], cannabinoid receptor 1 (CNR1) [74], neurexin 1 (NRXN1) [75], and neurotrophic tyrosine kinase receptor type 2 
Table 1. Candidate Genes Associated with Personality Traits for Psychiatric, Behavioral, and Substance-Related Disorders

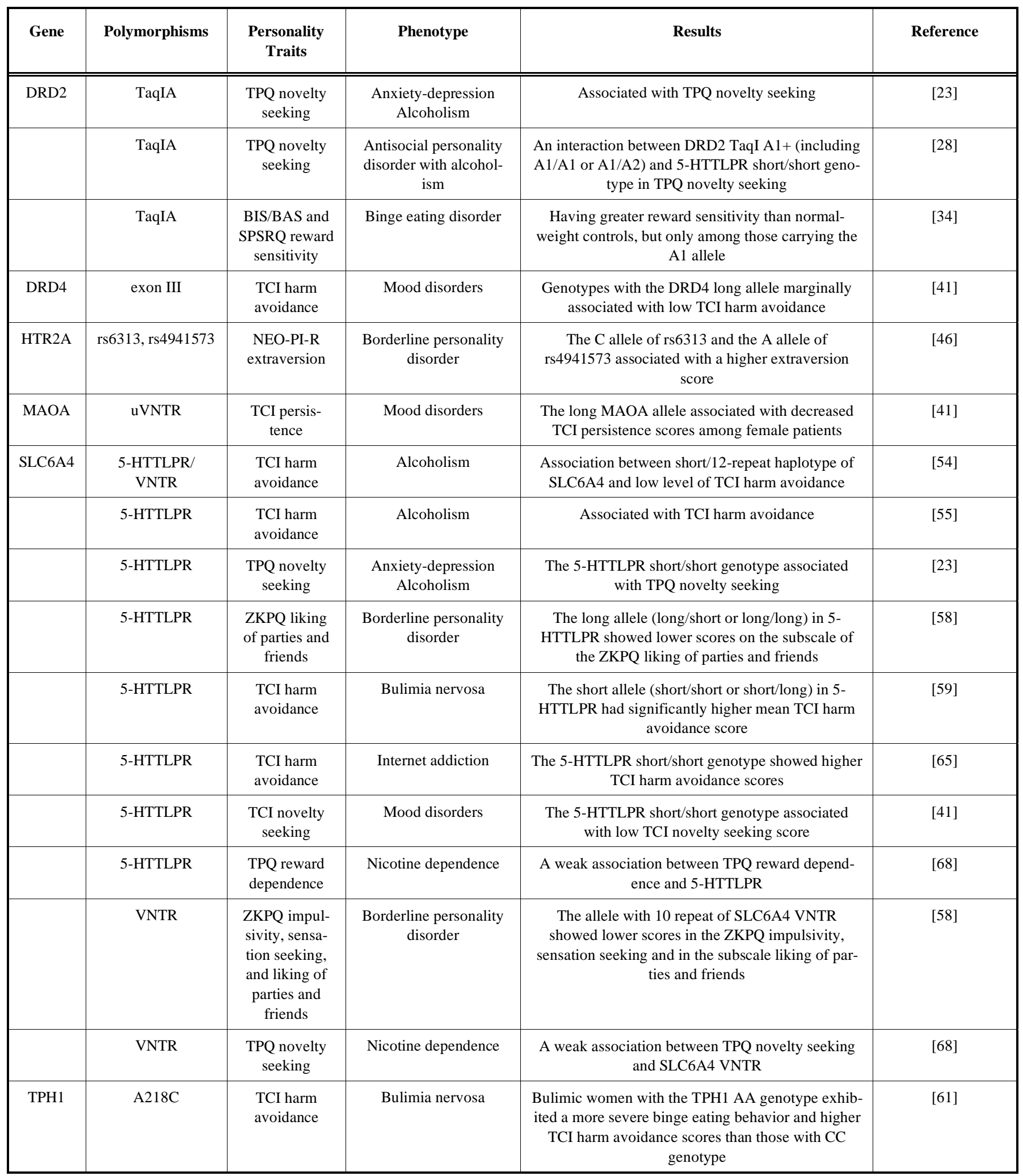

The "rs number" means the NCBI SNP ID.

5-HTTLPR, serotonin transporter gene-linked polymorphic region; BIS/BAS, Behavioral Inhibition System/Behavioral Activation System; DRD2, dopamine D2 receptor; DRD4, dopamine D4 receptor; HTR2A, 5-hydroxytryptamine receptor 2A (serotonin receptor 2A); MAOA, monoamine oxidase A; NEO-PI-R, Revised NEO Personality Inventory; SLC6A4, solute carrier family 6 member 4 (serotonin transporter); SPSRQ, Sensitivity to Punishment and Sensitivity to Reward Questionnaire; TCI, Temperament and Character Inventory; TPH1, tryptophan hydroxylase 1; uVNTR, the upstream variable number of tandem repeats in the MAOA gene promoter; VNTR, the variable number tandem repeats region in the second intron of the SLC6A4 gene; TPQ, Tridimensional Personality Questionnaire; ZKPQ, Zuckerman-Kuhlman Personality Questionnaire. 
(NTRK2) [76]. In future work, the contributions of these genes to personality traits in nicotine dependence should be further examined.

\section{CONCLUSIONS}

Table 1 summarizes the candidate genes associated with personality traits for psychiatric, behavioral, and substancerelated disorders as described in this article. This is by no means a comprehensive review of all potential markers reported in the literature. As mentioned previously, increasing numbers of markers are being identified as researchers continue to pay attention to personality genetics. As shown in Table 1, the 5-HTTLPR polymorphism of the SLC6A4 gene was found to be related to TCI harm avoidance for alcoholism, bulimia nervosa, and internet addiction $[55,59,65]$. Moreover, the SLC6A4 5-HTTLPR polymorphism was suggested to be linked to TPQ/TCI novelty seeking with anxiety-depression alcoholism and mood disorders [23,41]. In anxiety-depression alcoholism, it was also indicated that the DRD2 TaqIA and SLC6A4 5-HTTLPR polymorphisms were associated with TPQ novelty seeking [23]. In addition, the SLC6A4 and TPH1 genes seem to be involved in predisposing patients with bulimia nervosa to higher TCI harm avoidance $[59,61]$. Furthermore, the SLC6A4 and HTR2A genes may play a role in personality traits with borderline personality disorder [46,58]. By examining subjects in specific populations with psychiatric, behavioral, and substance-related disorders and testing association between candidate genes in these groups, these studies provide a favorable approach to solve the complexity problems of personality genetics [7].

With respect to the previously discussed association studies in the personality genetic study, several limitations include issues such as small effect sizes, polygenic inheritance, environmental influences, the constraints of self-report questionnaires, generalizability, population stratification, multiethnicity, studies without genome-wide association and considering gene-gene and gene-environment interactions [7].

In future work, it will be indispensably necessary to identify a panel of candidate genes that are reproducibly associated with personality traits for psychiatric, behavioral, and substance-related disorders. At this point, no genetic markers listed in the previous studies would really qualify to enter the panel due to the limitations as described above. There are several selection criteria for genetic markers to enter such a panel. One is independent replications in genome-wide association studies to confirm associations. It is now technically and financially feasible to conduct genome-wide association studies using dense genotyping chips [77,78]. Moreover, a multistage approach with independent samples is needed to replicate findings [77]. Finally, pattern recognition techniques such as the artificial neural network approach may provide a plausible way to assess gene-gene and geneenvironment interactions [79-81].

\section{ACKNOWLEDGEMENTS}

The authors extend their sincere thanks to Vita Genomics, Inc. for funding this research. This work was partially supported by the Department of Health, Taiwan (DOH96TD-D-113-041). The authors would also like to thank the anonymous reviewers for their constructive comments, which improved the context and the presentation of this paper.

\section{REFERENCES}

[1] Ebstein RP, Novick O, Umansky R, et al. Dopamine D4 receptor (D4DR) exon III polymorphism associated with the human personality trait of novelty seeking. Nat Genet 1996; 12: 78-80.

[2] Benjamin J, Li L, Patterson C, et al. Population and familial association between the D4 dopamine receptor gene and measures of novelty seeking. Nat Genet 1996; 12: 81-4.

[3] Lesch KP, Bengel D, Heils A, et al. Association of anxiety-related traits with a polymorphism in the serotonin transporter gene regulatory region. Science 1996; 274: 1527-31.

[4] Savitz JB, Ramesar RS. Genetic variants implicated in personality: a review of the more promising candidates. Am J Med Genet B Neuropsychiatr Genet 2004; 131: 20-32.

[5] Noblett KL, Coccaro EF. Molecular genetics of personality. Curr Psychiatry Rep 2005; 7: 73-80.

[6] Torgersen S. Behavioral genetics of personality. Curr Psychiatry Rep 2005; 7: 51-6.

[7] Ebstein RP. The molecular genetic architecture of human personality: beyond self-report questionnaires. Mol Psychiatry 2006; 11: 427-45.

[8] Cloninger CR. A systematic method for clinical description and classification of personality variants. A proposal. Arch Gen Psychiatry 1987 ; 44 : 573-88.

[9] Cloninger CR, Svrakic DM, Przybeck TR. A psychobiological model of temperament and character. Arch Gen Psychiatry 1993; 50: $975-90$.

[10] Costa Jr PT, McCrae RR. Stability and change in personality assessment: the revised NEO personality inventory in the year 2000 . J Pers Assess 1997; 68: 86-94.

[11] Eysenck HJ, Eysenck SBG, Barrett P. A revised version of the psychoticism scale. Pers Individ Dif 1985; 6: 21-9.

[12] Eysenck HJ, Eysenck SBG. Manual for the Eysenck Personality Questionnaire-Revised. San Diego, CA: Educ Ind Test Serv 1992.

[13] Zuckerman M, Kuhlman DM. Personality and risk-taking: common biosocial factors. J Pers 2000; 68(6): 999-1029.

[14] Sen S, Burmeister M, Ghosh D. Meta-analysis of the association between a serotonin transporter promoter polymorphism (5HTTLPR) and anxiety-related personality traits. Am J Med Genet B Neuropsychiatr Genet 2004; 127B(1): 85-9.

[15] Schinka JA, Busch RM, Robichaux-Keene N. A meta-analysis of the association between the serotonin transporter gene polymorphism (5-HTTLPR) and trait anxiety. Mol Psychiatry 2004; 9(2): 197-202.

[16] Munafò MR, Freimer NB, Ng W, et al. 5-HTTLPR genotype and anxiety-related personality traits: A meta-analysis and new data. Am J Med Genet B Neuropsychiatr Genet 2008 Jun 10 [Epub ahead of print].

[17] Hansenne M, Pinto E, Pitchot W, et al. Further evidence on the relationship between dopamine and novelty seeking: A neuroendocrine study. Pers Individ Dif 2002; 33(6): 967-77.

[18] Peirson AR, Heuchert JW, Thomala L, et al. Relationship between serotonin and the Temperament and Character Inventory. Psychiatry Res 2000; 89(1): 29-37.

[19] Garvey MJ, Noyes R, Cook B, et al. Preliminary confirmation of the proposed link between reward-dependence traits and norepinephrine. Psychiatry Res 1996; 65(1): 61-4.

[20] De Fruyt F, Van De Wiele L, Van Heeringen C. Cloninger's psychobiological model of temperament and character and the fivefactor model of personality. Pers Individ Dif 2000; 29: 441-52.

[21] Stallings MC, Hewitt JK, Cloninger CR, Heath AC, Eaves LJ. Genetic and environmental structure of the Tridimensional Personality Questionnaire: three or four temperament dimensions? J Pers Soc Psychol 1996; 70: 127-40.

[22] Girault JA, Greengard P. The neurobiology of dopamine signaling. Arch Neurol 2004; 61(5): 641-4.

[23] Lin SC, Wu PL, Ko HC, et al. Specific personality traits and dopamine, serotonin genes in anxiety-depressive alcoholism among Han Chinese in Taiwan. Prog Neuropsychopharmacol Biol Psychiatry 2007; 31(7): 1526-34.

[24] Huang SY, Lin WW, Ko HC, et al. Possible interaction of alcohol dehydrogenase and aldehyde dehydrogenase genes with the dopamine D2 receptor gene in anxiety-depressive alcohol dependence. Alcohol Clin Exp Res 2004; 28(3): 374-84.

[25] Hesselbrock VM, Hesselbrock MN. Are there empirically supported and clinically useful subtypes of alcohol dependence? Addiction 2006; 101(Suppl 1): 97-103. 
[26] Hovatta I, Barlow C. Molecular genetics of anxiety in mice and men. Ann Med 2008; 40(2): 92-109.

[27] Huang SY, Lin WW, Wan FJ, et al. Monoamine oxidase-A polymorphisms might modify the association between the dopamine D2 receptor gene and alcohol dependence. J Psychiatry Neurosci 2007; 32(3): 185-92.

[28] Wu CY, Wu YS, Lee JF, et al. The association between DRD2/ANKK1, 5-HTTLPR gene, and specific personality trait on antisocial alcoholism among Han Chinese in Taiwan. Am J Med Genet B Neuropsychiatr Genet 2008; 147B(4): 447-53.

[29] Fitzgerald KL, Demakis GJ. The neuropsychology of antisocial personality disorder. Dis Mon 2007; 53(3): 177-83.

[30] Moffitt TE. Genetic and environmental influences on antisocial behaviors: evidence from behavioral-genetic research. Adv Genet 2005; 55: 41-104.

[31] Livesley WJ, Jang KL. The behavioral genetics of personality disorder. Annu Rev Clin Psychol 2008; 4: 247-74.

[32] Mateu C, Haro G, Barabash A, et al. The role of genetics in the personality and its disorders: a clinical point of view. Actas Esp Psiquiatr 2008; 36(4): 230-43.

[33] Wang TJ, Huang SY, Lin WW, et al. Possible interaction between MAOA and DRD2 genes associated with antisocial alcoholism among Han Chinese men in Taiwan. Prog Neuropsychopharmacol Biol Psychiatry 2007; 31(1): 108-14.

[34] Davis C, Levitan RD, Kaplan AS, et al. Reward sensitivity and the D2 dopamine receptor gene: A case-control study of binge eating disorder. Prog Neuropsychopharmacol Biol Psychiatry 2008; 32(3): 620-8.

[35] Carver CS, White TL. Behavioral inhibition, behavioral activation, and affective responses to impending reward and punishment: The BIS/BAS Scales. J Pers Soc Psychol1994; 67(2): 319-33.

[36] Torrubia R, Ávilab C, Moltób J, et al. The Sensitivity to Punishment and Sensitivity to Reward Questionnaire (SPSRQ) as a measure of Gray's anxiety and impulsivity dimensions. Pers Individ Dif 2001; 31(6): 837-62.

[37] Striegel-Moore RH, Franko DL. Should binge eating disorder be included in the DSM-V? A critical review of the state of the evidence. Annu Rev Clin Psychol 2008; 4: 305-24.

[38] Berkman ND, Bulik CM, Brownley KA, et al. Management of eating disorders. Evid Rep Technol Assess (Full Rep) 2006; (135): 1-166.

[39] Monteleone P, Tortorella A, Castaldo E, et al. Association of a functional serotonin transporter gene polymorphism with binge eating disorder. Am J Med Genet B Neuropsychiatr Genet 2006; 141B(1): 7-9.

[40] Monteleone P, Tortorella A, Castaldo E, et al. The Leu72Met polymorphism of the ghrelin gene is significantly associated with binge eating disorder. Psychiatr Genet 2007; 17(1): 13-6.

[41] Serretti A, Mandelli L, Lorenzi C, et al. Temperament and character in mood disorders: influence of DRD4, SERTPR, TPH and MAO-A polymorphisms. Neuropsychobiology 2006; 53(1): 9-16.

[42] Kato T. Molecular genetics of bipolar disorder and depression. Psychiatry Clin Neurosci 2007; 61(1): 3-19.

[43] Savitz JB, Ramesar RS. Personality: is it a viable endophenotype for genetic studies of bipolar affective disorder? Bipolar Disord 2006; 8(4): 322-37.

[44] Levinson DF. The genetics of depression: a review. Biol Psychiatry 2006; 60(2): 84-92.

[45] Leysen JE. 5-HT2 receptors. Curr Drug Targets CNS Neurol Disord 2004; 3(1): 11-26.

[46] Ni X, Bismil R, Chan K, et al. Serotonin 2A receptor gene is associated with personality traits, but not to disorder, in patients with borderline personality disorder. Neurosci Lett 2006; 408(3): 214-9.

[47] Lieb K, Zanarini MC, Schmahl C, et al. Borderline personality disorder. Lancet 2004; 364(9432): 453-61.

[48] Lis E, Greenfield B, Henry M, et al. Neuroimaging and genetics of borderline personality disorder: a review. J Psychiatry Neurosci 2007; 32(3): 162-73

[49] Barnow S, Herpertz SC, Spitzer C, et al. Temperament and character in patients with borderline personality disorder taking gender and comorbidity into account. Psychopathology 2007; 40(6): 36978.

[50] Pinsonneault JK, Papp AC, Sadée W. Allelic mRNA expression of $\mathrm{X}$-linked monoamine oxidase a (MAOA) in human brain: dissection of epigenetic and genetic factors. Hum Mol Genet 2006; 15(17): 2636-49.
[51] Caspi A, Sugden K, Moffitt TE, et al. Influence of life stress on depression: moderation by a polymorphism in the 5-HTT gene. Science 2003; 301(5631): 386-9.

[52] Serretti A, Calati R, Mandelli L, et al. Serotonin transporter gene variants and behavior: a comprehensive review. Curr Drug Targets 2006; 7(12): 1659-69.

[53] Lin E, Chen PS. Pharmacogenomics with antidepressants in the STAR*D study. Pharmacogenomics 2008; 9(7): 935-46.

[54] Koller G, Zill P, Skoruppa T, et al. Low level of harm avoidance is associated with serotonin transporter functional haplotype in alcohol-dependent individuals. Psychiatr Genet 2008; 18(2): 59-63.

[55] Wiesbeck GA, Weijers HG, Wodarz N, et al. Serotonin transporter gene polymorphism and personality traits in primary alcohol dependence. World J Biol Psychiatry 2004; 5 (1): 45-8.

[56] Mayfield RD, Harris RA, Schuckit MA. Genetic factors influencing alcohol dependence. Br J Pharmacol 2008; 154(2): 275-87.

[57] Schumann G, Johann M, Frank J, et al. Systematic analysis of glutamatergic neurotransmission genes in alcohol dependence and adolescent risky drinking behavior. Arch Gen Psychiatry 2008; 65(7): 826-38.

[58] Pascual JC, Soler J, Baiget M, et al. Association between the serotonin transporter gene and personality traits in borderline personality disorder patients evaluated with Zuckerman-Zuhlman Personality Questionnaire (ZKPQ). Actas Esp Psiquiatr 2007; 35(6): 382-6.

[59] Monteleone P, Santonastaso P, Mauri M, et al. Investigation of the serotonin transporter regulatory region polymorphism in bulimia nervosa: relationships to harm avoidance, nutritional parameters, and psychiatric comorbidity. Psychosom Med 2006; 68(1): 99-103.

[60] Wang L, Erlandsen H, Haavik J, et al. Three-dimensional structure of human tryptophan hydroxylase and its implications for the biosynthesis of the neurotransmitters serotonin and melatonin. Biochemistry 2002; 41(42): 12569-74.

[61] Monteleone P, Tortorella A, Martiadis V, et al. Association between A218C polymorphism of the tryptophan-hydroxylase-1 gene, harm avoidance and binge eating behavior in bulimia nervosa. Neurosci Lett 2007; 421(1): 42-6.

[62] Hay PJ. Understanding bulimia. Aust Fam Physician 2007; 36(9): 708-12, 731.

[63] Ando T, Komaki G, Naruo T, et al. Possible role of preproghrelin gene polymorphisms in susceptibility to bulimia nervosa. Am J Med Genet B Neuropsychiatr Genet 2006; 141B(8): 929-34.

[64] Beard KW. Internet addiction: a review of current assessment techniques and potential assessment questions. Cyberpsychol Behav 2005; 8(1): 7-14

[65] Lee YS, Han DH, Yang KC, et al. Depression like characteristics of 5HTTLPR polymorphism and temperament in excessive internet users. J Affect Disord 2008; 109(1-2): 165-9.

[66] Munafò MR, Clark TG, Roberts KH, et al. Neuroticism mediates the association of the serotonin transporter gene with lifetime major depression. Neuropsychobiology 2006; 53(1): 1-8.

[67] Markou A. Review. Neurobiology of nicotine dependence. Philos Trans R Soc Lond B Biol Sci 2008; 363(1507): 3159-68.

[68] Kremer I, Bachner-Melman R, Reshef A, et al. Association of the serotonin transporter gene with smoking behavior. Am J Psychiatry 2005; 162(5): 924-30.

[69] Beuten J, Ma JZ, Payne TJ, et al. Significant association of BDNF haplotypes in European-American male smokers but not in European-American female or African-American smokers. Am J Med Genet B Neuropsychiatr Genet 2005; 139: 73- 80.

[70] Lang UE, Sander T, Lohoff FW, et al. Association of the met66 allele of brain-derived neurotrophic factor (BDNF) with smoking. Psychopharmacology (Berl) 2007; 190: 433-9.

[71] Li MD, Beuten J, Ma JZ, et al. Ethnicand gender-specific association of the nicotinic acetylcholine receptor alpha4 subunit gene (CHRNA4) with nicotine dependence. Hum Mol Genet 2005; 14 : 1211-9.

[72] Feng Y, Niu T, Xing H, et al. A common haplotype of the nicotine acetylcholine receptor alpha 4 subunit gene is associated with vulnerability to nicotine addiction in men. Am J Hum Genet 2004; 75 : 112-21.

[73] Hutchison KE, Allen DL, Filbey FM, et al. CHRNA4 and tobacco dependence: From gene regulation to treatment outcome. Arch Gen Psychiatry 2007; 64: 1078 -86.

[74] Chen X, Williamson VS, An SS, et al. Cannabinoid receptor 1 gene association with nicotine dependence. Arch Gen Psychiatry 2008; 65(7): 816-24. 
[75] Nussbaum J, Xu Q, Payne TJ, et al. Significant association of the neurexin-1 gene (NRXN1) with nicotine dependence in Europeanand African-American smokers. Hum Mol Genet 2008; 17(11): 1569-77.

[76] Beuten J, Ma JZ, Payne TJ, et al. Association of specific haplotypes of neurotrophic tyrosine kinase receptor 2 gene (NTRK2) with vulnerability to nicotine dependence in African-Americans and European-Americans. Biol Psychiatry 2007; 61: 48-55.

[77] Shifman S, Bhomra A, Smiley S, et al. A whole genome association study of neuroticism using DNA pooling. Mol Psychiatry 2008; 13(3): 302-12.
[78] Heck A, Lieb R, Unschuld PG, et al. Evidence for associations between PDE4D polymorphisms and a subtype of neuroticism. Mol Psychiatry 2008; 13(9): 831-2.

[79] Lin E, Hwang Y, Liang KH, Chen EY. Pattern-recognition techniques with haplotype analysis in pharmacogenomics. Pharmacogenomics 2007; 8(1): 75-83.

[80] Lin E, Hwang Y, Chen EY. Gene-gene and gene-environment interactions in interferon therapy for chronic hepatitis C. Pharmacogenomics 2007; 8(10): 1327-35.

[81] Lin E, Hsu SY. A Bayesian approach to gene-gene and geneenvironment interactions in chronic fatigue syndrome. Pharmacogenomics 2009; 10(1): 35-42.

Received: December 03, 2008

(C) Lin and Chen; Licensee Bentham Open.

This is an open access article licensed under the terms of the Creative Commons Attribution Non-Commercial License (http://creativecommons.org/licenses/by-nc/3.0/) which permits unrestricted, non-commercial use, distribution and reproduction in any medium, provided the work is properly cited. 